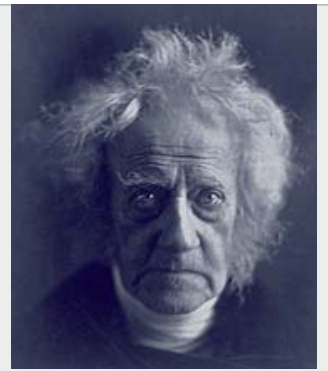

\section{Principales dates}

7 mars 1792 - Naissance à Slough (Buckinghamshire, Angleterre)

1825 Médaille Lalande de l'Académie des Sciences de Paris

1830 Discourse on Natural Philosophy

1833 Royal Medal de la Royal Society

11 mai 1871 - Décès à Hawkhurst (Kent, Angleterre)

\title{
John Frederick William
}

\section{Herschel}

\section{Riad Haidar}

haidar@onera.fr

Astronome illustre, mathématicien talentueux et chimiste doué, John Frederick William Herschel est connu pour ses contributions fondamentales à la photographie, et son apport séminal sur le concept de Philosophie Naturelle qui inspirera toute une génération de grands esprits britanniques, dont Darwin et Faraday.

${ }^{7}$ ohn Frederick William Herschel naît le 7 mars 1792 à Slough, dans le comté du Buckinghamshire, de l'union de Mary Baldwin, fille d'un marchand prospère, et du fameux William Herschel [1738-1822]. William est issu d'une famille de musiciens allemands; il a immigré à 19 ans en Angleterre comme garde hanovrien. Hautboïste de formation, compositeur de métier, il est surtout passionné d'astronomie, qu'il pratique tardivement et $d^{\prime}$ abord en amateur, avant d'inventer la science stellaire : on lui doit notamment la découverte d'Uranus en 1781 et la première véritable carte de l'Univers. Mary, veuve Pitt, épouse William en secondes noces, et le couple est déjà âgé à la naissance de John. II sera leur seul enfant.

\section{Une enfance studieuse}

Le jeune garçon grandit dans la maison de l'observatoire, un lieu magique près du château de Windsor, dans une ambiance rare qui mêle ferveur religieuse, inspiration musicale et effervescence scientifique. Enfant unique, John bénéficie de l'attention éclairée de tous, et notamment de sa tante Caroline Herschel, immigrée en 1772, qui seconde son père dans ses recherches et fréquente assidûment l'observatoire. Elle s'avère une tutrice exceptionnelle, initiant son neveu aux mystères de la physique et de la chimie.

Le moment venu, John ne parvient pas à se détacher de cet univers singulier et à s'adapter à l'école. À huit ans, il fréquente l'école du Dr Gretton à Hitcham, puis le collège d'Eton. Mais il est harcelé (semble-t-il) par ses camarades et, au bout de quelques mois, sa mère décide de le retirer du circuit scolaire. Dès lors, il étudie à domicile sous la houlette de Mr Rogers, qui lui enseigne les mathématiques, et de sa tante Caroline. II se forge ainsi une solide culture scientifique et est admis au Collège St John de l'Université de Cambridge en 1809.

II se lie d'amitié avec ses camarades de promotion Peacock [1791-1858] et Babbage [1792-1871], avec lesquels il fonde en 1813 l'Analytical Society. Ils ont l'objectif d'introduire en Angleterre les outils d'analyse développés en Europe continentale, notamment la notation différentielle et le calcul intégral de d'Alembert, Leibniz et Lagrange.

Cette même année John, étudiant très actif et particulièrement fécond, est élu fellow de la Royal Society de Londres, et achève son cursus à Eton en senior
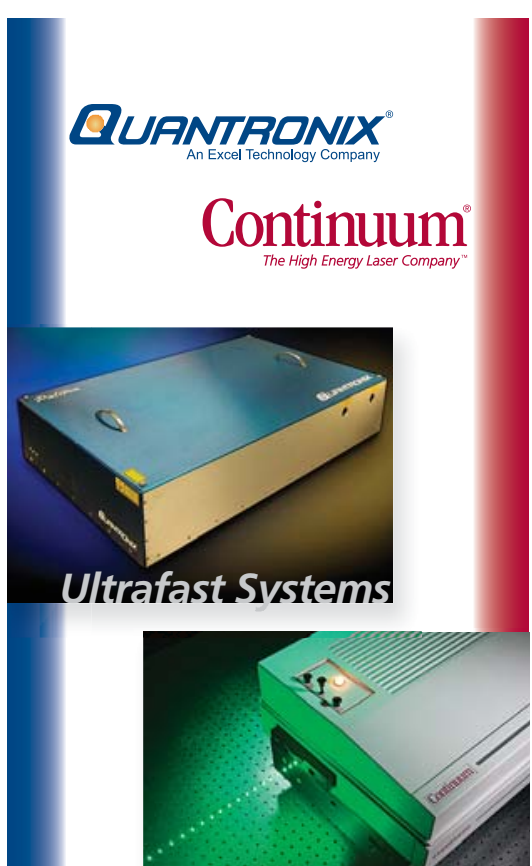

Hioh Enerorv YAG.

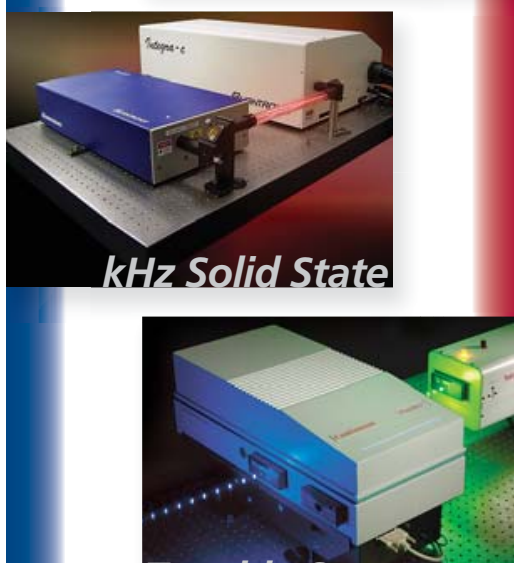

Tunable Systems

Innovative Laser Solutions for Science and Industry

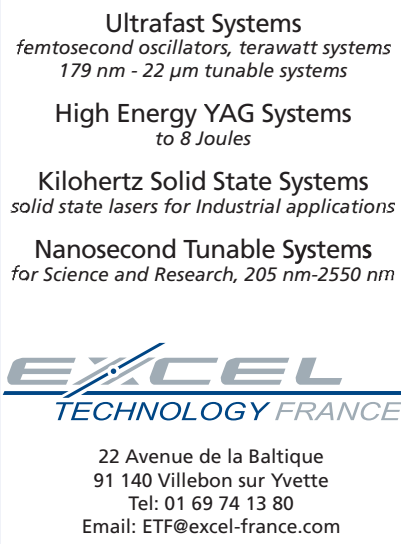

Ultrafast Systems femtosecond oscillators, terawatt systems $179 \mathrm{~nm}-22 \mu \mathrm{m}$ tunable systems High Energy YAG Systems to 8 Joules

Kilohertz Solid State Systems solid state lasers for Industrial applications

Nanosecond Tunable Systems for Science and Research, 205 nm-2550 nm

TECHNOLOGY FRANCE

22 Avenue de la Baltique 91140 Villebon sur Yvett Tel: 0169741380 Email: ETF@excel-france.com

Serving France for over 25 years 
wrangler (major de promotion). La voie semble alors tracée vers les mathématiques; éventuellementen soutane, comme le souhaite son père... Pourtant, Herschel décide de se former au droit. II dissout I'Analytical Society, et s'inscrit à Londres. $C^{\prime}$ est une parenthèse surprenante, qui ne lui convient guère à l'usage et qu'il referme finalement au bout de 18 mois.

\section{Vers I'astronomie}

En 1815, il retourne à Cambridge, et aux mathématiques. II obtient une bourse Smith, et est nommé fellow du Collège St John. Il publie quelques travaux mineurs, et surtout un livre sur les différences finies, récompensé par la Copley Medal de la Royal Society en 1821. Mais son esprit en ébullition l'attire bien vite loin de l'univers éthéré des mathématiques pures.

En 1816, il passe les mois d'été avec son père, alors âgé de 78 ans, à Slough. $C^{\prime}$ est une formation accélérée au métier délicat d'astronome : il polit un miroir de 18 pouces et assemble son propre télescope, puis se perfectionne à l'observation stellaire... Dès lors, plus ou moins consciemment, mais irrésistiblement, John se destine à l'astronomie.

Quelques années plus tard, abandonnant ses autres activités, notamment des travaux en chimie qui s'avéreront 20 ans plus tard d'un intérêtfondamental pour les premiers procédés photographiques, il entreprend de poursuivre l'œuvre de son père. En effet celle-ci, bien que féconde et originale, menace de s'éteindre faute de successeur. Endossant l'illustre costume des Herschel, John s'implique lourdement dans la création de l'Astronomical Society, qui voit enfin le jour en 1820 et dont il est élu vice-président.

Secondé de James South [17851867], il complète en 1824 le catalogue des étoiles doubles initié par William, décédé en 1822. Ce travail considérable est couronné par la Médaille Lalande de I'Académie des Sciences de Paris en 1825, et par la Gold Medal de la Royal Astronomical Societyen 1826. Mais I'ambition est ailleurs : il veut utiliser la parallaxe des étoiles pour en déduire la distance à la Terre. C'est une méthode prometteuse et Herschel fait des avancées considérables, récompensées par la Royal Medal en 1833. Elle s'imposera en 1838 avec les résultats spectaculaires obtenus par Bessel [1784-1846] sur la Binaire 61 de la constellation du Cygne.

\section{Voyage en Afrique du Sud}

Dans les années 1820, Herschel voyage fréquemmenten Europe Continentale, où il fait la connaissance d'Arago [1786-1853], Fraunhofer [1787-1826] ou Laplace [1749-1827], autant de rencontres qui façonnent son appréhension de la science. II s'investit également davantage dans la vie scientifique londonienne : il est nommé secrétaire de la Royal Society en 1824 et en manque de peu la présidence en 1831 ; il est élu président de l'Astronomical Society en 1827. En 1830, John Herschel publie son fameux traité, Discourse on Natural Philosophy, où il fonde la méthode de l'investigation scientifique, qui inspirera Faraday [1791-1867], Darwin [1809-1882], et toute une génération de grands esprits britanniques. II rédige ou fait insérer plusieurs mémoires et articles dans des recueils et encyclopédies. Signalons en particulier son article On the Theory of Light publié en 1831, où il défend I'hypothèse d'une lumière ondulatoire.

Il épouse Margaret Brodie Stewart [1810-1884] en 1829 à Edimbourg. En novembre 1833, le couple s'embarque avec leurs trois enfants (ils en auront douze) pour l'Afrique du Sud. John s'installe au Royal Observatory du Cap de Bonne-Espérance, fraîchement inauguré en 1828. II souhaite étudier les objets célestes qui ne sont pas observables depuis I'hémisphère Nord. II met ainsi au point des techniques inédites de photométrie pour calibrer la clarté des étoiles, et permettre une comparaison objective avec celles du ciel boréal.

La famille, qui se plaît dans sa résidence de Feldhausen, au pied de la Montagne de la Table, prolonge son séjour jusqu'en 1838. Et Herschel multiplie les observations, notamment celle de la comète de Halley lors de son passage en 1835. II réunira l'ensemble de ses résultats en 1847 dans le volumineux Cape Observations, qui lui vaut sa seconde Copley Medal de la Royal Society.

\section{Photographie}

À son retour en Angleterre, John Herschel, qui a été anobli en 1831, est fait Baronnet de Slough du Comté de Buckinghamshire lors du couronnement de la Reine Victoria en juin 1838. En janvier 1839, il découvre fortuitement les travaux de Daguerre [1787-1851] sur la photographie, un art et une science qui se sont développés pendant son absence d'Europe. S'inspirant de ses premiers travaux en chimie, il mène rapidement ses propres expériences et invente la technique de fixation (par le désormais classique hyposulfite de sodium) des images sur leur support.

Les honneurs, de même que les charges, s'accumulent. II est nommé recteur du Marischal College d'Aberdeen en 1842. 1 est élu à l'Académie Royale de Suède en 1836, et membre associé de l'Académie des Sciences de Paris en 1855, au fauteuil de Gauss [1777-1855]. En 1850, Herschel accepte la prestigieuse, mais lourde fonction de Master of the Mint llittéralement, maître de la monnaie), qui exige des déplacements fréquents dans tout le royaume et des tractations financières et politiques qui l'épuisent. II en démissionne en 1855 pour raison de santé et se retire à Collingwood, sa résidence près de Hawkhurst dans le Kent. Pour autant il reste très actif, et publie en 1864 un catalogue général des nébuleuses et des amas, qui est une compilation de ses propres observations et de celles de son père William.

II s'éteint le 11 mai 1871, à l'âge de 79 ans. II est inhumé à l'Abbaye de Westminster, à proximité du caveau qui abrite les restes de Newton [1642-1727]. En raison de sa vaste activité intellectuelle et pédagogique, et de ses contributions originales etfécondes en astronomie, mathématiques et photographie, il est reconnu et toujours célébré en Angleterre comme l'une des grandes figures scientifiques de son temps, à l'instar de Laplace en France.

\section{Référence}

G. Buttman, "The Shadow of the Telescope: A Biography of John Herschel" (Lutterworth Press). 